Document downloaded from:

http://hdl.handle.net/10251/45033

This paper must be cited as:

Ghafoor, KZ.; Lloret, J.; Abu Bakar, K.; Sadiq, AS.; Ben Mussa, SA. (2013). Beaconing Approaches in Vehicular Ad Hoc Networks: A Survey. Wireless Personal Communications. 73(3):885-912. doi:10.1007/s11277-013-1222-9.

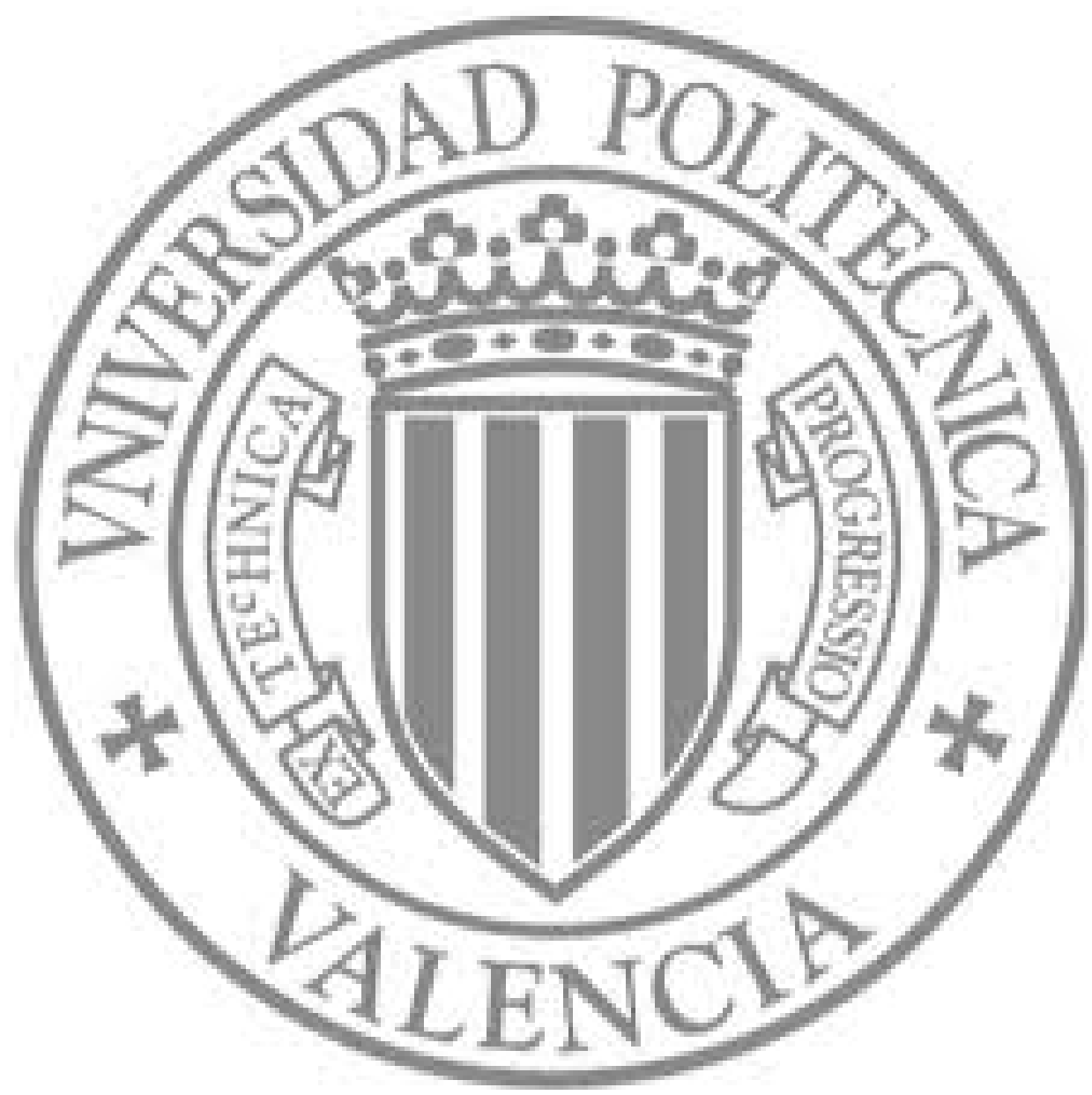

The final publication is available at

http://dx.doi.org/10.1007/s11277-013-1222-9

Copyright Springer Verlag (Germany) 


\title{
Beaconing Approaches in Vehicular Ad Hoc Networks: A Survey
}

\author{
Kayhan Zrar Ghafoor · Jaime Lloret · Kamalrulnizam \\ Abu Bakar · Ali Safa Sadiq · Sofian Ali Ben Mussa
}

the date of receipt and acceptance should be inserted later

\begin{abstract}
A Vehicular Ad hoc Network (VANET) is a type of wireless ad hoc network that facilitates ubiquitous connectivity between vehicles in the absence of fixed infrastructure. Beaconing approaches is an important research challenge in high mobility vehicular networks with enabling safety applications. In this article, we perform a survey and a comparative study of state-of-the-art adaptive beaconing approaches in VANET, that explores the main advantages and drawbacks behind their design. The survey part of the paper presents a review of existing adaptive beaconing approaches such as adaptive beacon transmission power, beacon rate adaptation, contention window size adjustment and Hybrid adaptation beaconing techniques. The comparative study of the paper compares the representatives of adaptive beaconing approaches in terms of their objective of study, summary of their study, the utilized simulator and the type of vehicular scenario. Finally, we discussed the open issues and research directions related to VANET adaptive beaconing approaches.
\end{abstract}

Keywords V2V Communications - Adaptive Beaconing - Transmission Power - Beacon Rate . Contention Window

\section{Introduction}

Recently, the growth in the number of vehicles on the road has put great stress on transportation systems. This abrupt growth of vehicles has made driving unsafe and hazardous. Thus, existing transportation infrastructure requires improvements in traffic safety and efficiency. To accomplish this, Intelligent Transportation Systems (ITS) have been considered to enable such diverse traffic applications as traffic safety, cooperative traffic monitoring and control of traffic flow. These traffic applications would become realities through the emergence of VANET because it is considered as a network environment of ITS. Moreover, the range of applications is increasing daily. The increasing necessity of this network is an impetus for leading car manufacturers, research communities and governments to increase their efforts toward creating a standardized platform for vehicular communications. In particular, the $5.9 \mathrm{GHz}$ spectrum band has been allocated for licensed Short Range Communication (DSRC)

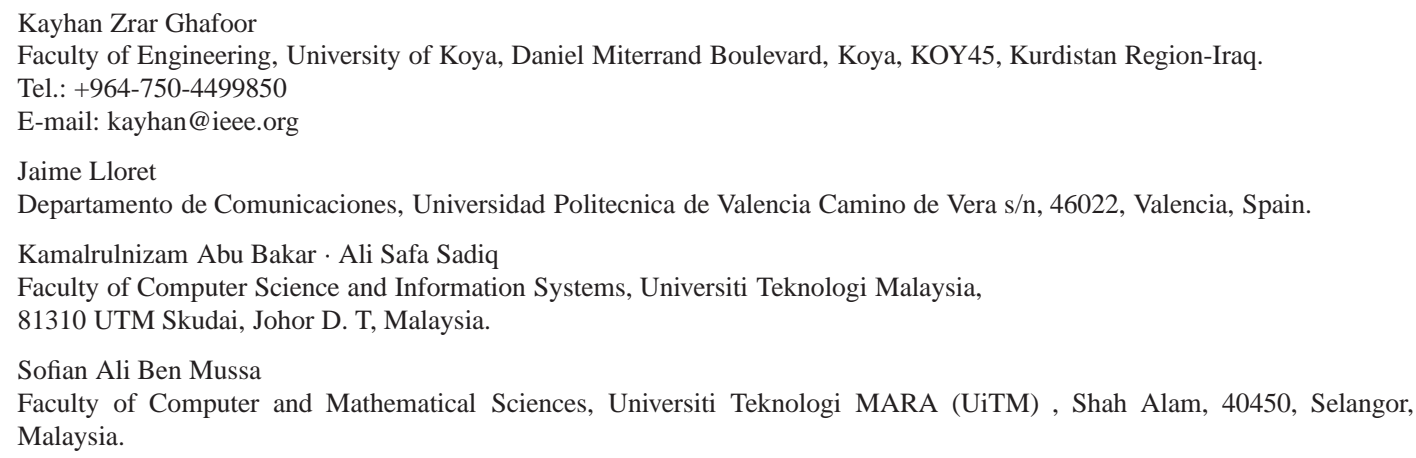




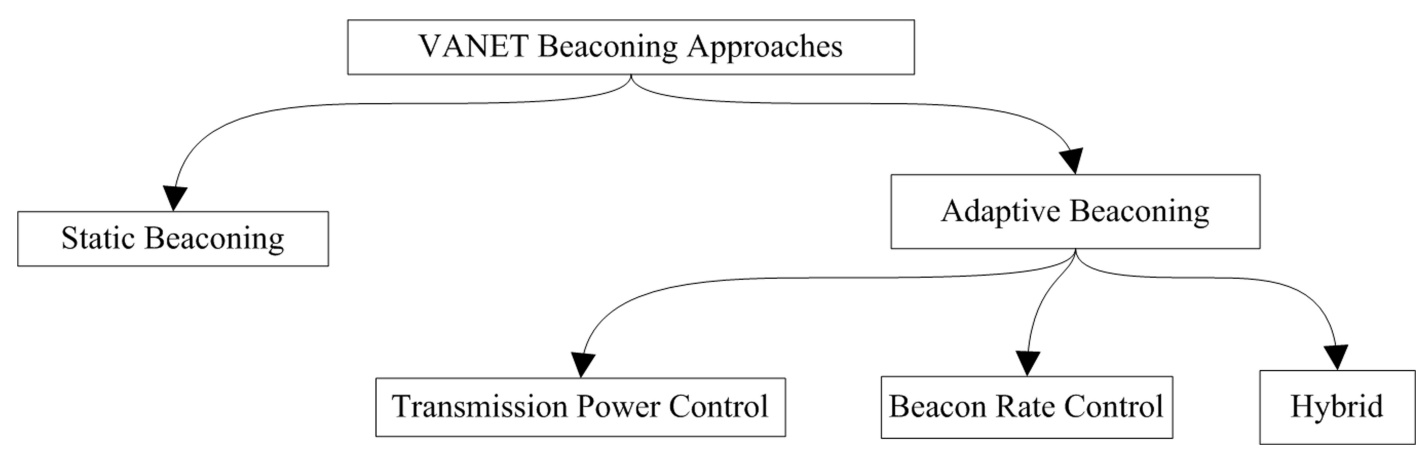

Fig. 1: Taxonomy of the literature on beaconing approaches.

between vehicles. In addition, in the near future, more vehicles will be embedded with devices that facilitate communication between vehicles, such as IEEE 802.11p, called Wireless Access in Vehicular Environment (WAVE) [1]. The WAVE wireless technology is extended from the IEEE standard of 802.11a and adopted service differentiation of IEEE 802.11e to support multimedia applications. When vehicles are equipped with WAVE, they can synchronize and handshake via beacons. In this way, a vehicle exchanges beacon messages periodically, sharing its mobility characteristics with its neighbours, thereby building cooperative awareness.

The main purpose of mobile ad hoc networks (MANET) is to maximize lifetime and capacity of the wireless networks. In contrast to MANET, vehicular networks are emerged to improve traffic safety related applications through beacon (periodic) and event-driven messages [2]. Beacon messages are crucial to enable safety applications. Each vehicle broadcasts periodic beacon messages to inform neighbour nodes about their address, location and other relevant information. This status messages can be used to detect abnormal situation on the highway and urban scenarios. On the other hand, dangerous situations like car accidents will trigger event-driven messages to be disseminated with highest priority to nearby vehicles.

In this proactive neighbourhood awareness, each vehicle should maintain the up-to-date list of neighbour nodes. Otherwise, the outdated information problem of the neighbour list leads to miss the next candidate node or the node that has been chosen will move out the radio range.

However, rapid changes in traffic density from sparse to heavy, as well as periodic beaconing between vehicles, can cause the wireless channel between vehicles to promptly become congested, resulting in a high degree of performance degradation of vehicular network [3], [4], [5]. The reason for this channel congestion is that each vehicle periodically broadcasts beacons at a fixed rate. This also leads to high channel overloading and hence packet loss. In short, the higher the frequency of beacon rate, the higher the bandwidth overload in dense traffic conditions [6].

On the other hand, the solution to channel overloading does not involve simply reducing the frequency of beacon generation. As the frequency of beacon generation is reduced, the error will increase between the current physical position and the last reported position. For instance, in geographical routing protocols, reducing beacon rate would lead to the inaccuracy of the exchanged position coordinates between vehicles. This would negatively affect the performance of routing protocols. In short, reducing the beacon rate leads to the exchange of out-of-date information. To tackle this issue, the authors in [7], [8], [9], [10], [11] [12] considered conditional update approach in which a vehicle adapts its beacon rate based on various parameters.

In addition, due to fast movement of vehicles link life time will last very soon in VANET. Increasing radio coverage of the vehicle is counted as a method of increasing the link life time when vehicles are sparsely deployed. But, the increase of transmission range might negatively affect the VANET performance when vehicles are densely deployed. This is due to higher interference and overhead. Thus, dynamic transmission power adaptation is an imperative need in such bipolar traffic distribution environment [13], [14]. On the other hand, quality of service differentiation of various applications are very important in VANET since vehicles might expose to different incidents [15]. 
In this article, a concise description of the background of beaconing approaches will be presented first, followed by an elaboration of the main types of adaptive beaconing approaches, and the differences between them. The overall taxonomy of beaconing approaches are depicted in Fig. 1. Then, the classification of each type of VANET's beaconing approaches will be demonstrated and their implementation are explored. That is, adaptation of beacon transmission power with respect to the heterogeneous vehicular traffic distribution, adjustment of beacon rate and hybrid adaptive beaconing approaches. After that, it provides a survey of recent state of the art beaconing approaches, the motivation behind their design and trace the influence of mobility model on these beaconing approaches. At the end of the paper, we summarize the beaconing approaches as shown in Table 1. We finally conclude this paper by discussing important open issues and research directions in VANET.

\section{Beaconing Approaches}

Cooperative awareness demands up-to-date and low delay information exchange between vehicles. This information includes position, movement and acceleration of the vehicles in the vicinity. This can be achieved by broadcasting beacon messages. Recently, European ITS system in [16] has proposed a fixed beaconing approach which requires every vehicle to establish its cognizance about other nearby vehicles. Furthermore, Cooperative Active Safety System(CASS) utilizes information exchanged between neighbour vehicles in order to provide assistance to drivers. The CASS system may successfully provide this awareness by transmitting vehicular information, at a fixed rate through beacon message, every $100 \mathrm{~ms}$ in all vehicular scenarios $[17,18]$. This vehicular awareness supports geographical routing and data dissemination. However, since the beacon message size is approximately 400 bytes [19], it requires a profound amount of bandwidth as traffic density increases, leading performance deterioration in the network [20], [21], [22], [23]. Fig. 2 shows a vehicular scenario where the source vehicle disseminates the beacon message to nearby vehicles.

The problem of beaconing adaptation has been studied in various prospects in VANET. Transmission power control and beacon rate control are two main examples of adaptation approaches. The authors in [4], [24], [25], [20], [26], [15], [27] have proposed adaptation approaches to tune transmission power with varying vehicular densities. That is, the purpose is to reduce transmission power in dense vehicular scenarios and hence improve fairness. In addition, adaptation of beaconing can be done by controlling the beacon rate with heterogeneous distribution of vehicles.

For the purpose of tuning the contention window size, Van Eenennaam et al. in [22] proposed a framework to adapt network and MAC-layer parameters in order to mimic the configuration parameters. This adaptive approach can tune MAC layer configurations and beaconing properties to optimal values in the vehicular scenarios. The authors demonstrate the requirement of efficient beaconing approach to increase cooperative awareness between vehicles. Fig. 3 shows that increased distance leads to a lack of cooperative awareness. However, vehicular networks are dynamic, as evidenced by dense rush hours and sparse late night traffic conditions. In designing their model, the aforementioned authors did not take these factors into consideration.

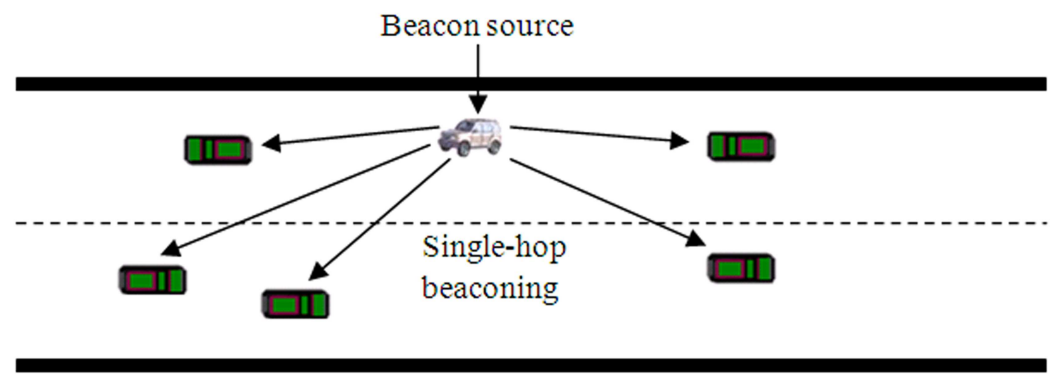

Fig. 2: Illustration of single-hop beacon dissemination 


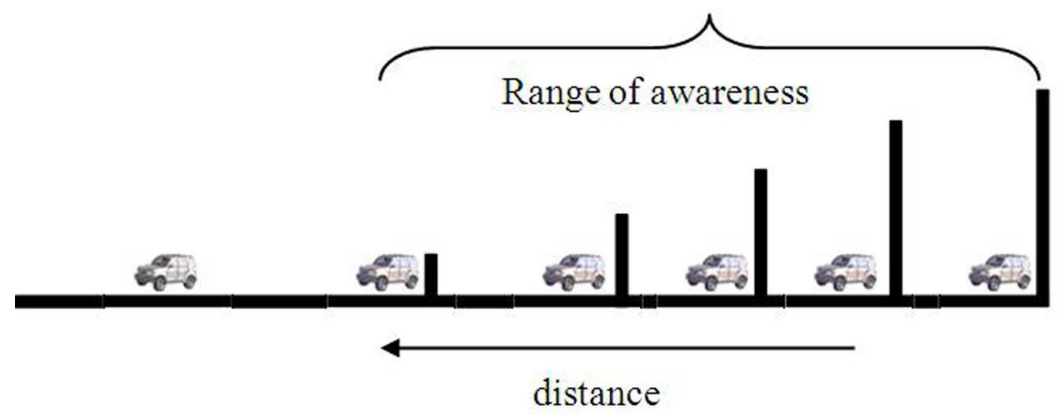

Fig. 3: Cooperative awareness between vehicles

\section{Adaptive Beaconing Approaches}

\subsection{Beacon Transmission Power Control}

As mentioned in previous section, periodic and even-driven messages are required to activate safety applications. Periodic beacon messages are necessary to enable awareness of nearby vehicles by exchanging state information such as position, speed, direction and other vehicle's information. This beaconing message handshaking captures important information of vehicles in the vicinity and thereby each vehicle will be able to detect emergency situations in the surroundings. The beaconing load on the wireless channel should precisely be monitored to guarantee bandwidth requirement of active safety applications. To this purpose, the authors in [28] conducted performance analysis of active safety related applications in order to identify mechanisms to improve VANET performance through reducing packet level interference. For this purpose, they proposed a distributed fair transmit power adjustment algorithm for vehicular ad Hoc networks called D-FPAV. It maintains the beaconing load so that the active safety applications meet a strict deadline of the event driven message. In addition, they developed a fast beacon message dissemination towards destination using receiver based relay node self election.

D-FPAV limits the channel overhead on the wireless medium due to periodic beacon message transmission and provides the entire channel capacity in case of the occurrence of any emergency situations. The priority of message transmission is achieved through EDCA of the IEEE 802.11p. In other words, event driven messages have highest priority and maximum transmit power as compared to the periodic beacon message. For the fast dissemination of beacon safety messages, the authors in [4, 29] proved desirable and satisfactory performance of beaconless geographical forwarding. According to this forwarding mechanism, the emergency message is transmitted to all neighbour nodes and they independently elect themselves as a next forwarder. The proposed mechanism favours farthest node to be a next beacon message forwarder.

The performance evaluations were done using a popular Network Simulator (ns-2) [30]. Although ns-2 is a widely used network simulator among researchers, there are some defects in terms of accuracy of lower layers. To tackle this issue, the authors added a cumulative noise module to the physical layer of the IEEE 802.11p. A node accumulates the power level of all interfering packets that generated from neighbour nodes with the background noise.Then, the node decides whether the beacon message is successfully received. The probabilistic Nakagami [31] propagation model was used since it can estimate the fading phenomena of wireless channel in vehicular scenario with buildings and trees. In the performance evaluation, the D-FPAV On is compared with the D-FPAV Off. It is worth mentioning that with D-FPAV Off all beacon messages are sent with maximum Carrier Sense range $(\mathrm{CS}=1000 \mathrm{~m})$ whereas in D-FPAV On the beacon transmission power is computed based on the DFPAV algorithm. Results show that D-FPAV On always performs better than when it is compared to D-FPAV Off in terms of probability of successful packet reception and channel access time.

The aims of transmission range adaptation in mobile wireless networks are to increase throughput, network and link lifetime $[13,32]$. These conventional objectives of power control can not be applicable in vehicular networks since network topology and communication between vehicles have 


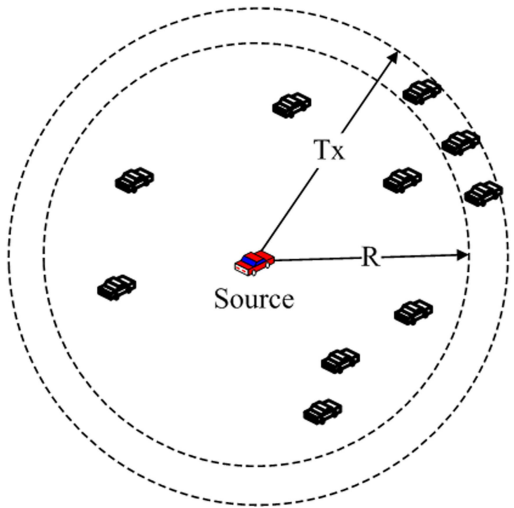

(a) Tx is larger than $\mathrm{R}$.

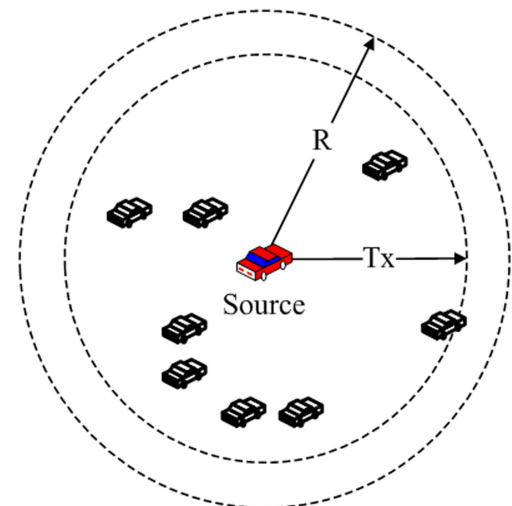

(b) $\mathrm{R}$ is larger than $\mathrm{Tx}$.

Fig. 4: Effect of variable target and transmission range on the power control.

unique characteristics when they are compared with traditional mobile networks. In addition, vehicleto-vehicle communication is emerged to improve traffic safety on the road. Thus, power control in vehicular networks should fulfill reliability and delay sensitive active safety applications. This interesting application has motivated some researchers to develop suitable power control mechanism. For instance, the authors in [13] proposed a transmission power control algorithm of safety messages that are exchanged between nearby vehicles. The algorithm is based on the fact that each vehicle periodically broadcast its status information and the required information for power control is piggybacked in the safety message header. The piggybacked information is the target range and feedback beacon. The target range is defined as the specified range up to which the transmitted safety message is effective.

As depicted in Fig.4, vehicles are traveling along a highway with three lanes. Vehicle effective communication range and target range are denoted as $\mathrm{Tx}$ and $\mathrm{R}$ respectively. The source node first broadcasts a safety message to nearby vehicles. When the neighbour vehicle received the message, the relative distance is computed between the message sender and receiver. The source node realizes that the Tx range is larger than $\mathrm{R}$ range based on the feedback from the neighbour nodes that are outside the $\mathrm{R}$ range. In this case, the transmission power will be reduced (Fig.4a). If this case is not happen, the transmission power of the safety message is increased by the source (Fig.4b).

The scheme proposed by the authors in [13] is simulated on the OPNET network simulator with modifying 802.11 a to model 802.11p MAC layer. The log-normal radio propagation model is used to model slow fading in which the wireless channel is not susceptible to fast variation during communication between vehicles. Simulation results show that the proposed scheme performs better as compared to a scheme with no power control on terms of probability of message loss. However, the performance of proposed power control scheme is not optimal since the response packet from neighbour receivers might be lost due to channel collisions and leads to higher transmit power by the source node.

Following the trend of adjusting the transmission power of packet carrier vehicles, the authors in [33] proposed an adaptive power control mechanism based traffic and propagation modeling. Lognormal shadowing is used to model the power propagation in highway vehicular scenario and real highway data has been used to model vehicular traffic distribution from late night to the daytime rush hours. Performance evaluation results of the proposed mechanism is compared with the no-power control algorithms in three traffic situations: early morning, moderate morning traffic and evening rush hour. Results show that the expected transmission power reduces from the early morning to the evening rush hour.

As witnessed by the authors in [34] and [35], a dynamic adaptation of transmission range is important in heterogeneous traffic distribution like vehicular networks. This improves the network performance in terms of packet collisions, interference and network overhead. To tackle the connectivity issues in such bipolar traffic environment, the authors in [36] proposed a mechanism to adapt vehicles 


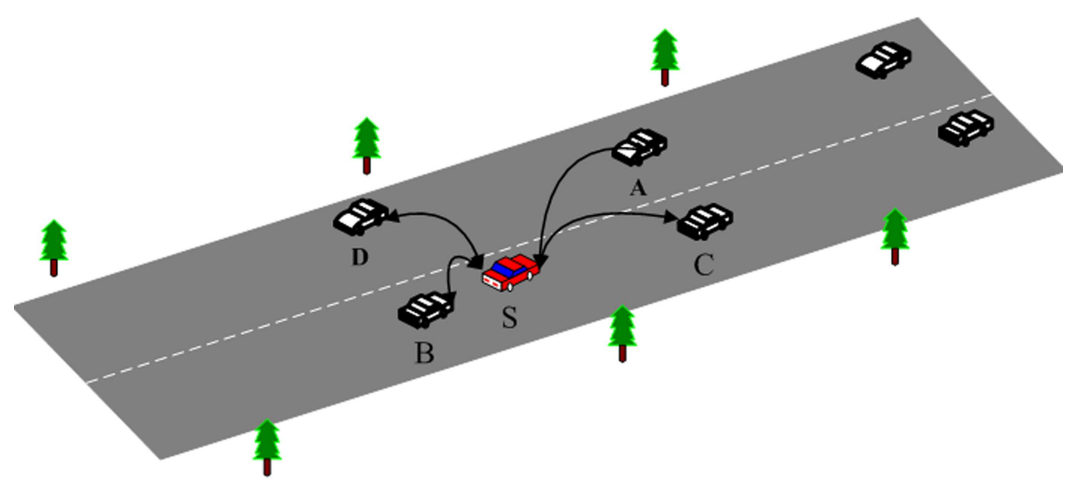

Fig. 5: Vehicle S receives beacon messages from neighbour vehicles.

transmission range based on local vehicles traffic density estimation. The traffic density estimation is based on the movement of vehicles i.e., when a vehicle travels with high speed, the local density is low and vice versa. Simulation results shown in this paper reveal that the proposed mechanism maintains the link life time of the wireless channels between high speed vehicles.

In another attempt of dynamic power transmission, Samara et al. in [37] proposed a scheme based on the numerical analysis to control the transmission power of the periodic beacon message. The basic idea of the proposed scheme is that the a vehicle counts the number of beacons lost or successfully received in 1 second from the neighbouring vehicles. As depicted in Fig.5, if the beacon generation rate is $10 \mathrm{~ms}$, vehicle $\mathrm{S}$ must receive 10 beacon messages from each neighbour vehicle (A,B,C,D) every 1 second. More precisely, the proposed scheme calculates the scheduled transmission power as in Equ.1:

$$
T P=\operatorname{Min} P+(P D \times P S)
$$

where TP is the new transmission power, MinP is minimum power of neighbour vehicles (this parameter is included into beacon message), PD is the power difference between maximum and minimum received power of neighbour vehicles and PS is the percentage of success of beacon message reception. When a vehicle (such as $\mathrm{S}$ in Fig.5) receives a beacon message extracts minimum and maximum received power from them. Moreover, PD as well as PS can be determined based on the number of beacon messages that are received successfully.

\subsection{Beacon Rate Control}

As we have aforementioned, high beacon rate leads to network congestion while low beacon rate causes inaccurate shared position information. To tackle this problem, the authors in [38] and [39] considered adaptation of beacon rate in vehicular environment. The proposed beacon rate adaptation is based on differences in predicted positions. In their prediction scheme, all vehicles are embedded with modified Kalman estimators to provide continuous estimations of existing positions. This position estimate can be obtained via the last beacon message, enhancing positional accuracy between two sequential beacons. Moreover, the prediction scheme requires that the next beacon message is triggered based on a vehicle's current position and an estimated position. Once the vehicle determines a change in its physical position, it triggers the next beacon message. In this way, vehicles independently estimate the duration of the next beacon message. However, rapid topology changes of vehicles and mobility traffic characteristics were not considered.

Fukui et al. in [7] proposed a beacon adaptation scheme which considers the distance travelled by vehicles. Moreover, vehicles independently determine the number of lanes the current road has, and the higher the number of lanes, the lower the beacon rate. In addition, another beacon adaptation technique is based on packet loss rate. But, changing beacon rates based on multi lanes is unfair 
because multi lanes do not directly imply higher traffic density. Further, the accuracy of information has not been considered.

Authors in [8] first studied the adaptation of beacon rate in order to find the compromise between information accuracy and bandwidth consumption. After the analysis of the parameters, which affect to the beacon rate, they proposed a scheme to adapt beacon rate according to the VANET traffic behaviour. In their study, however, intelligently combined traffic parameters like direction, density and status of a vehicle have been neglected. Moreover, their study is based on theoretical analysis.

In our previous research [9], we proposed an intelligent Adaptive (situation-aware) Beaconing Rate (ABR) approach based on fuzzy logic to control the frequency of beaconing by taking traffic characteristics into consideration. The proposed ABR considers the percentage of vehicles travelling in the same direction, and status of vehicles as inputs of the fuzzy decision making system, in order to tune the beaconing rate according to the vehicular traffic characteristics. For comparison purpose with fixed beaconing rate approach, the ABR approach is implemented in JIST/SWANs. The simulation results show that the proposed ABR approach is able to improve channel load due to beaconing, improve cooperative awareness between vehicles and reduce average packet delay in lossy/lossless urban vehicular scenarios.

The authors in [11] modelled and simulated multi-hop and single hop beaconing in vehicular networks. The aim of the study is to address the fundamental question whether the beaconing load on the wireless channel can be reduced if periodic beacon messages are transmitted over multiple hops with reduced transmitted power instead of being transmitted over single hop with high transmit power. In their study, they noted that the multi-hop beaconing is necessary in situations where non-line-ofsight area obstruct a radio range by single hop beaconing.

In [12], Sommer et al. proposed Adaptive Traffic Beacon (ATB) approach to achieve congestion aware and low latency wireless channel. The ATB approach is a broadcast-based approach that precisely designed to use the residual capacity of wireless channel. In particular, the proposed solution is adaptive in the sense that the beacon generation rate is changed dynamically with respect to the channel quality and importance of the message as well as their solution can dynamically use infrastructure networks in the vicinity. Simulation results show that the ATB maintains a non-congested wireless channel to prevent packet collisions during the data exchange. In another attempt, the authors in [40] extended their ATB in order to compare it with flooding-based approaches. Simulation experiments demonstrate that the proposed ATB achieves broader coverage than flooding-based approaches, but at the expenses of transmission rate.

The above approaches $[12,40]$ did not consider the effect of MAC layer settings on beacon reception probability and delay. The authors in [19] investigated the effect of MAC layer Contention Window (CW) on the efficiency of IEEE 802.11 p beacon broadcasts. More precisely, their analysis improves the performance of the $802.11 \mathrm{p}$ broadcasts in terms of reception probability and delay with a focus on real-time vehicle control.

Because vehicles are travelling in a very high speed, network topology is continuously changing along the time. In this harsh environment, vehicles could communicate and synchronize through exchanging of beacon messages. To promote situation-aware beacon communication between vehicles, adaptive beaconing is necessary in order to reduce network overhead. In [41], Thaina et al. proposed two methods to adapt beacon rate with frequent topology changing of vehicular networks. The first method is linear regression analysis which can be represented as follows:

$$
\widehat{Y}=a+b X
$$

where $\mathrm{a}$ and $\mathrm{b}$ are regression coefficients, which are computed in equ. 3 and equ. 4 :

$$
a=\bar{y}-b \bar{x}
$$

$$
b=\frac{\sum_{a=1}^{m}\left(x_{i}-\bar{x}\right) \times\left(y_{i}-\bar{y}\right)}{\sum_{a=1}^{m}\left(x_{i}-\bar{x}\right)^{2}}
$$




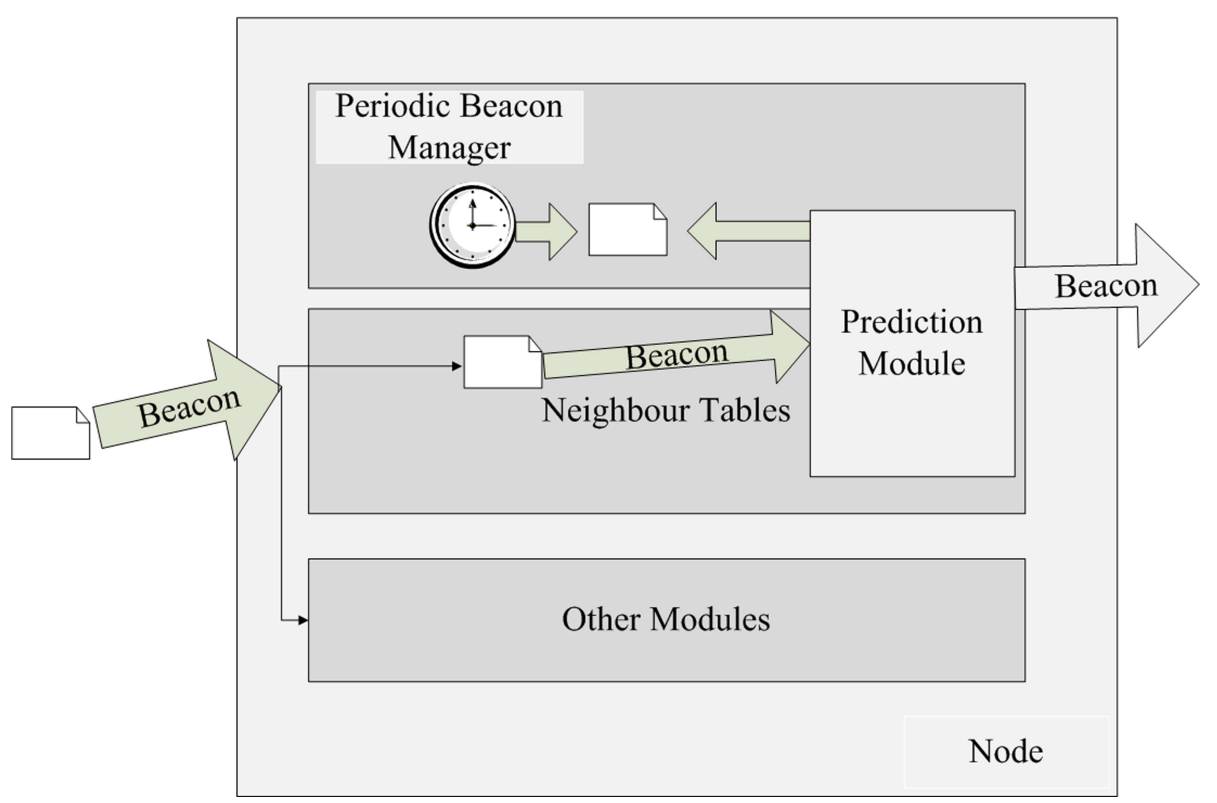

Fig. 6: Periodic beacon trigger and module interaction within a node

where $\bar{x}$ and $\bar{y}$ are the average value of $\mathrm{x}$ and $\mathrm{y}$ respectively. As a result, each vehicle counts number of neighbour nodes and queued messages to estimate network traffic and density. The value of $\mathrm{V}$ is calculated as the summation of these two parameters. After this the value of $\mathrm{X}$ is obtained. Then, it is substituted in equ.2. From equ.2, we can compute the value of $\widehat{Y}$ which is the next beacon interval.

Their second method for estimating next beacon interval is $k$-nearest neighbour which is used to calculate the approximate value of target function. In this method, each vehicle creates a table which contains the parameters of node's environment condition (network density and traffic) and beacon interval.

As mentioned early, vehicles usually moves at very high speed. The positional information exchanged between nearby vehicles promptly becomes outdated. The simple method to tackle this issue is to increase the transmission rate of beacon message transmission. But, this method might generate high traffic overhead in the network. To tackle this issue, the authors in [42] proposed an improved neighbour localization scheme that predicts the location of neighbours in the near future. Besides the node's current location, neighbour nodes use additional information stored in the beacon such as node's previous location, speed and direction. With these positional information, a vehicle uses predicted position of a neighbour vehicle instead of the position that exists in the received beacon. Each vehicle tracks the distance between actual and predicted position of the neighbour vehicle in order to define a threshold $\delta$. New beacon will be scheduled for transmission if the difference between predicted and actual position is greater than $\delta$. Fig. 6 shows the information flow and beacon rate scheduling inside a node. Next beacon message is scheduled in two cases: due to large error threshold between actual and predicted vehicle position, and periodically with constant rate.

In [42], the authors conducted an extensive simulation study of the proposed localization scheme in realistic highway scenario (Freeway) [43]. The Freeway simulation model is modified to incorporate vehicle overtaking in multi-lane vehicular scenario. The proposed solution, jointly with the ones presented in the state of the art, are evaluated based upon the parameters of mean error and number of transmitted beacons. Simulation results show that proposed localization algorithm dramatically increases the accuracy of localization of neighbour nodes and number of beacons sent as compared to the naive approach of beaconing. However, the proposed scheme is evaluated in unrealistic vehicular environment since radio obstacles are not considered in the simulation scenarios. In addition, only beacon transmission in single hop are considered in the devised localization scheme. 


\subsection{Hybrid Beaconing Approaches}

The basic principle of vehicle to vehicle communication is to improve the vehicle perception and predict the information of the other vehicles in the vicinity. This perception can be achieved through beaconing. In the near future, many VANET applications will rely on beaconing to enhance information sharing. In safety related applications, vehicles broadcast beacon message, which includes position, direction, velocity and VANET related information, to neighbour vehicles. However, the performance network might be deteriorated in terms of excessive packet delay and unreliability of beacon message dissemination in case of using naive beacon broadcasting service. To alleviate the problem of simple broadcasting, three key parameters can be adapted to vehicular network scenarios; beacon generation rate, beacon transmission power and contention window of the Medium Access Control (MAC) layer [44], [45], [15].

The authors in [45] proposed a beaconing congestion solution due to periodic beacon transmission that consumes large amount of available bandwidth resources. The proposed solution consists of three phases. The first phase assigns different levels of priority to the received beacon messages in order to differentiate their importance. When a vehicle receives a beacon message from nearby vehicles, its MAC layer ranks them based on the content of the messages and the hop count. The content of beacon messages are classified into immediate danger notification, warning message and driving information announcement. A vehicle receives more than one safety related messages and should assign priority to each message based on their contents. If the content of the messages have the same priority, the hop count should be considered in priority of message transmission. The priority of the message is inversely proportional with hop count metric.

The network is considered as congested if the number of packets in the network is higher than its processing capacity. In this case, the performance of the network is deteriorated due to high packet drops. As a consequence, the authors considered congestion detection mechanism in the second phase of the proposed solution. In VANET, congestion can be detected based on the continuous monitoring of the following metrics:

- Medium Busy Time (MBT): It is defined as the period in which the wireless medium (control channel, $\mathrm{CCH}$ ) is occupied due to transmissions from other vehicles in the vicinity.

- Packet Collision Rate: It is the ratio of unsuccessful transmissions from a node to total number of packets that are sent by the same node over the control channel.

- Beacon Reception Rate: This metric is defined as the ratio of number of beacons received from single hop neighbour to the total number of received beacons.

When the network is congested, a vehicle can control the beaconing load by adjusting its transmission power and beaconing generation rate. The transmitted power can be adapted based on the minimum transmission power used by the vehicle itself and vehicles in the vicinity and distance between the vehicle to next packet forwarder of the warning message. At the same time, a vehicle can adapt its beacon generation rate by equ. 5

$$
b=\frac{(B F)-B(\text { emergency }))}{B_{\text {size }}}
$$

where BF is the bandwidth fair share and B(emergency) is the estimated bandwidth that is enough to emergency related situations and $B_{\text {size }}$ denotes the beacon size. Fig.7 shows the general overview of the proposed beacon adaptation solution.

The performance evaluation was implemented using a OPNET [46] and in $10 \mathrm{~km}$ bidirectional four lane road section. In the simulation scenario, vehicles are travelling with a speed of 60 to $120 \mathrm{~km} / \mathrm{h}$ and 10 to 6 vehicle $/ \mathrm{km} / \mathrm{lane}$. Results of the simulation show that the proposed solution possesses higher beacon delivery ratio and emergency message reception as well as lower total delay as compared to only power control or beacon rate control solutions. Thus the combined power and beacon rate control is promising solution as compared to the existing solutions. However, in this study obstacle modelling is an imperative need to verify and validate the practical emergency message dissemination.

Since most of active safety applications require optimal broadcast of beacon frame in order to maintain accurate positional information while avoiding control channel congestion, the authors in [47] 


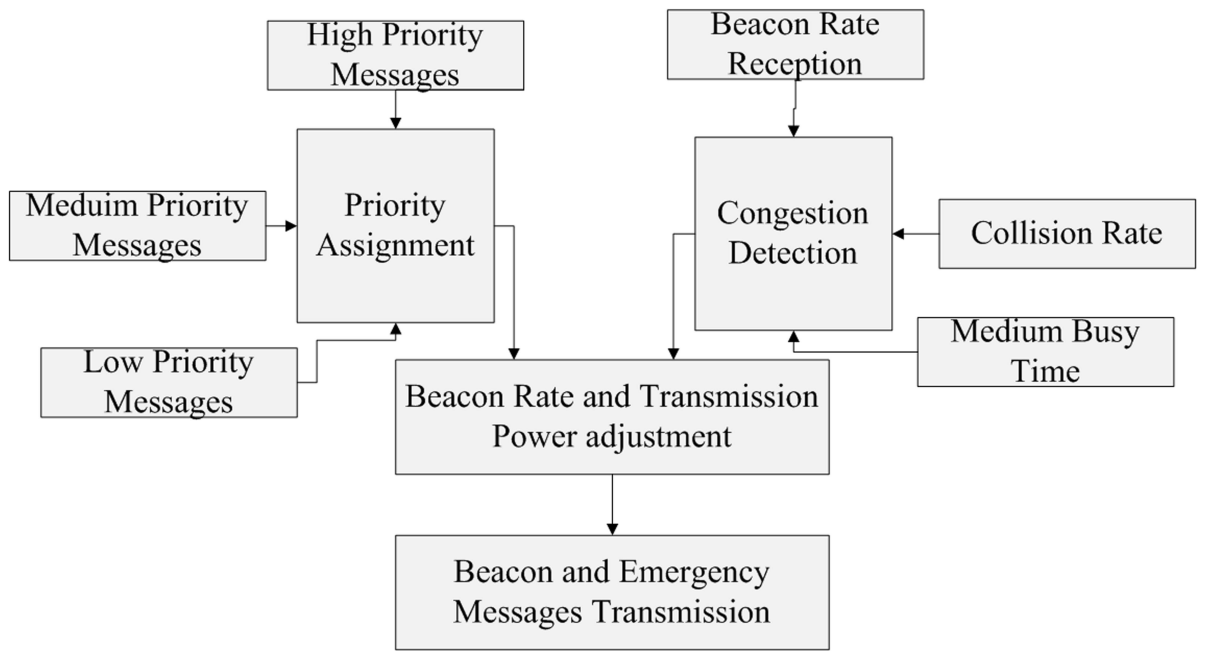

Fig. 7: Congestion detection and beacon adaptation schemes

propose a transmission control algorithm that controls beacon and transmission power rate based on the tracking accuracy and high speed topology variation of vehicular networks. The proposed algorithm considers closed loop control and error-prone and instability of wireless channels between vehicles. As can be observed in Fig.8, each vehicle embedded with communication control and position estimators track vehicles in the vicinity and plant measurements (to generate a vehicles state information). The proposed algorithm is implemented in the communication control section in each vehicle.

To control beacon transmission rate, the communication control logic of each vehicle tracks the error $e_{j}(t)$ difference between immediate vehicles toward its own position. Each vehicle computes the transmission probability $p_{j}(t)$ at time step $t$ from the value of tracking error. The algorithm first defines an error threshold $e_{t h}$ to control the transmission of beacon message, i.e., if the tracking error is greater than the error threshold, vehicle $j$ do not transmit a beacon message. In the other words, the value of $p_{j}(t)$ is equal to 0 and the control channel can be used by nearby vehicles. If this condition

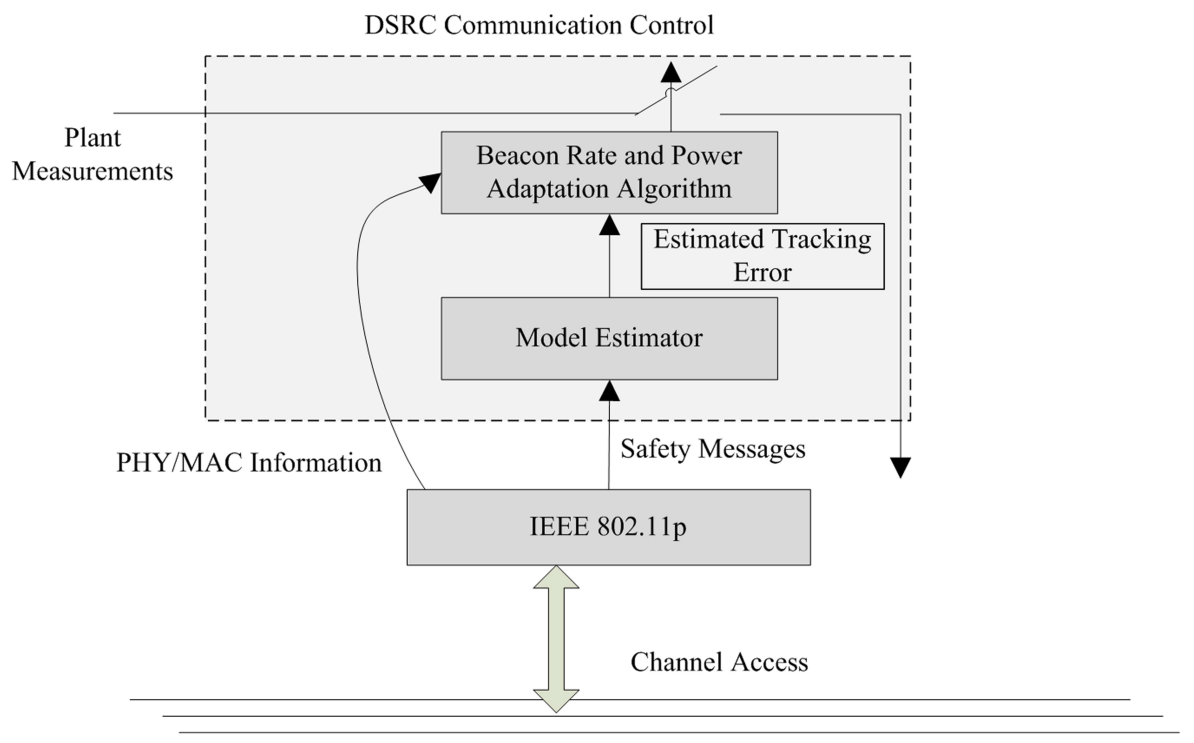

Fig. 8: Communication control and position estimator in each vehicle 
is not satisfied (i.e., $e_{j}(t) \geq e_{t h}$ ), vehicle $j$ calculates the probability of transmission by the following equ.6:

$$
p_{j}(t)=1-\exp \left(-\alpha \times\left|e_{j}(t)-e_{t h}\right|\right)^{2}
$$

where $\alpha$ denotes a sensitivity of the measured tracking error and its value is greater than 0 .

The authors also uses transmission power control to reduce beaconing load. In their mechanism, they used channel occupancy $U_{j} t$ as measure to tune transmission range of a vehicle. The value of $U_{j} t$ is between 0 to 1 and can be determined by Clear Channel Assessment (CCA) indicator of 802.11 MAC layer. This report acts as an indication by the PHY to the local MAC entity of the current state of the medium through PHY-CCA.indication (STATE) signal. If the wireless medium is busy of vehicle $j$, the PHY-CCA.indication (busy) returns to MAC layer otherwise the PHY-CCA.indication (idle) will be retuned to the MAC layer. Thus, the adapted transmission range at the time window $t$ can be defined in equ. 7

$$
L_{j}(t)=L_{\min }+\frac{U_{\max }-U_{j}(t)}{U_{\max }-U_{\min }} \times\left(L_{\max }-L_{\min }\right)
$$

where $L_{\min }$ and $L_{\max }$ are minimum and maximum transmission ranges, the values of these parameters depends on the application. Moreover, $U_{\max }$ and $U_{\min }$ are denotes minimum and maximum channel occupancy which are determined empirically.

The proposed mechanism is evaluated using OPNET [48] network simulator and realistic microscopic traffic simulator. Simulation results proves the robustness and less tracking error of the proposed algorithm in comparison with existing solutions (such as static beaconing interval of $100 \mathrm{~ms}$ and $500 \mathrm{~ms}$ ).

In another attempt, Nguyen et al. in [44] thoroughly analysed the impact of beacon rate, beacon transmission power and contention window on reducing beacon load in the wireless control channel. They first calculated the optimal beacon period to provide active safety applications without control channel congestion. Thus, the beacon period is defined as follows:

$$
b=\frac{D_{t h}}{v}
$$

where $D_{t h}$ is position error between two vehicles and $\mathrm{v}$ is the velocity of a vehicle. For the beacon transmit power control, the authors derived the vehicle density which is inversely proportional with the vehicle speed, i.e.,

$$
\lambda=\frac{1}{D_{I V}}=\frac{1}{D_{v}+\tau_{r} v+\frac{v^{2}}{2 a_{b}}}
$$

where $D_{I V}$ is the distance between two consecutive vehicles in the same road, $D_{v}$ is the vehicle length, $\tau_{r} v$ is the displacement distance due to vehicle perception-reaction, $\frac{v^{2}}{2 a_{b}}$ is the distance during braking-deceleration. As can be seen, the vehicle density decreases with the increase of vehicle speed. Thus, the velocity of vehicles and traffic density are implicitly interrelated to one another. This relationship is clearly known in traffic flow theory. In [49], Kerner states that the vehicular traffic density decreases as a result of increasing vehicle's average speed.

Each vehicle broadcast its won beacon in the Carrier Sensing Range (CSR). In the CSR of a vehicle the channel load due to beaconing can be computed as a multiplication of beacon load $\left(\frac{L}{B P}\right)$ of each vehicle with the traffic density within the CSR $\left(2 D_{C S R} K \lambda\right)$. Thus, beacon channel load within the CSR:

$$
C_{b}=\frac{2 D_{C S R} K \lambda}{D_{I V} D_{t h}}
$$

where $\mathrm{L}$ is the beacon size, $\mathrm{BP}$ is the beacon period and $D_{C S R}$ is the CSR. Thus, since the beacon load is proportional to the CSR, the beacon transmission power can be adjusted to reduce the CSR of the beacon. The active safety messages should be disseminated reliably and within acceptable time delay in the control channel. However, each vehicle uses control channel to disseminate beacon 
messages to the vehicles within its radio range. This beacon frequent broadcasting might occupy the control channel and thereby leads to congestion. In order to alleviate this situation, the occupation of control channel should be restricted to fraction $(0 \geq \alpha \leq 1)$ of the total wireless control channel (C) as shown in equ.11:

$$
C_{b}=\alpha C
$$

The maximum CSR $\left(D_{C S m a x}\right)$ to meet the channel requirement can be computed in equ.12 by combining equ. 11 and equ.10

$$
D_{C S \max }=\frac{\alpha C D_{I V} D_{t h}}{2 L K v}=\frac{\alpha C D_{t h}\left(D_{v}+\tau_{r} v+\frac{v^{2}}{2 a_{b}}\right)}{2 L K v}
$$

Following the trend, the authors in [15] proposed a joint dynamic adjustment of transmission power and contention window size. The adaptation of transmission power by first computing communication range and then using that range to determine the power level. The transmission range is calculated based on the vehicle's local traffic density as follows:

$$
C R=\min \left(L(1-K), \sqrt{\frac{L \ln L}{K}}+\alpha L\right)
$$

where $\alpha$ is the constant depends on the traffic flow, $\mathrm{L}$ is the length of each road segment, $\mathrm{CR}$ is the communication range, $\mathrm{K}$ is the local traffic density (i.e., is calculated as a ratio of actual number of vehicles over total number of vehicles within the transmission range of a vehicle). Consider vehicles with 300 meters of transmission range, which are travelling in two lanes highway (two lanes in each direction) in a regular road length of 700 meter. The safety distance between two consequent vehicles is 16 meters. For this highway configuration, the total number of vehicles is computed as $\frac{700}{16} \times 2 \times 2=$ 175 and we assume that the actual number of vehicles is 100 . From these values we can calculate $\mathrm{K}$ as $\frac{100}{175}=0.571$. Based upon this value and using equ.13, vehicles adjust their transmission range accordingly. After obtaining the value of transmission range, the proposed algorithm established a lookup table that maps the transmission power value to its equivalent transmission range.

In order to classify traffic-related messages, their proposed algorithm uses IEEE 802.11e EDCA mechanism in VANET. IEEE 802.11e EDCA consists of four access categories for prioritize incoming messages: voice traffic, video traffic, best effort traffic and background traffic. These Access categories $(\mathrm{AC}(0)$ to $\mathrm{AC}(3))$ have different channel access parameters. For instance, $\mathrm{AC}(3)$ has higher priority of message transmission in comparison to other access categories as it has lower minimum Contend Window $\left(C W_{\text {min }}\right)$. In such way, the VANET related messages are prioritized in order to assign highest priority to critical emergency messages.

The proposed algorithm is simulated in network simulator ns-2 and compared with the standard IEEE 802.11e EDCA protocol in terms of message delay and network throughput. Results show that the proposed algorithm reduces the end-to-end delay and increases average throughput as compared to the default IEEE 802.11e EDCA protocol.

\section{Static Beaconing Approaches}

The authors in [50] proposed an analytical model to calculate the probability of message reception by the packet's receiver and mean packet delay in IEEE $802.11 \mathrm{p}$ based vehicular networks. The proposed analytical model considers deterministic beaconing rate fashion rather than Poisson arrival pattern. This attribute of the devised protocol gives uniqueness in comparison to the state of the arts. They also evaluated the proposed analytical model on Matlab simulation model with 802.11p MAC layer. In their simulation, it is observed that the required time delay of a safety-related message can meet the threshold. However, the probability of beacon message reception could not meet the requirement value for critical applications. For instance, beacon message reception probability is 0.7 to 0.5 when Bit Error Rate is 0.0001.

Following the trends, Mariyasagayam et al. in [51] devised a multi-hop beaconing mechanism that adaptively controls the number of redundant messages during packet dissemination process. The 


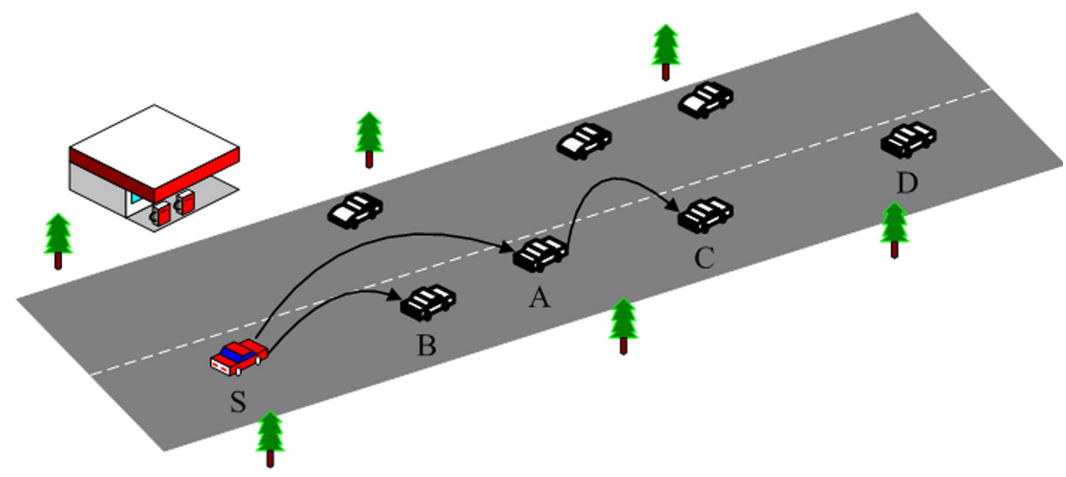

Fig. 9: Cooperative awareness between vehicles

algorithm divides the area falls within the communication range of the source to dynamic backfire sectors. Each sector is dynamic and depends upon the nodes density of nearby the packet source. This adaptive behaviour of each sector suppresses the broadcast of redundant messages. Fig. 9 depicts forwarding of the proposed mechanism where node $\mathrm{S}$ would broadcast packet to nearby vehicles (A and B). After receiving the packet, nodes A and B set the waiting time which inversely proportional with the distance between the source $(\mathrm{S})$ and packet receiver (A and B). In this scenario, node A broadcasts the packet since its timer expires first. After the node B receives the same packet form $S$ and $\mathrm{A}$, it drops the packet and thereby node A backfired node B.

The performance evaluation is based on the packet level simulator ns-2 [30] in urban vehicular environments. Results show that the proposed dynamics backfire mechanism performs better as compared to constant and no backfire mechanisms of packet forwarding in terms of packet delivery ratio. While radio signals are obstructed by obstacles in urban area, they used unrealistic radio propagation model, which is two ray ground model.

\section{Heterogeneous Vehicular Networks}

The applications of vehicular network are commonly categorized into two different groups: public safety and comfort-related applications. The undergoing studies in VANET only consider single type of applications. In the other words, none of them could achieve the requirements of all types of safety, traffic information system and comfort applications at the same time. Thus, it is necessary to enable a heterogeneous vehicular network in order to let a vehicle use multiple radio access technologies, i.e., (Worldwide Interoperability for Microwave Access) WiMAX, WiFi, IEEE 802.11p, $3 \mathrm{G}$ cellular networks, Long Term Evolution (LTE) and satellite communication.

The authors in [52], [53], [54], [55], [56] have studied the integration of ad hoc network with infrastructure to achieve the public safety and comfort-related applications. In [52], Hung et al. proposed mobility pattern-aware routing protocol for VANET. In this study, vehicles are equipped with IEEE 802.16e and IEEE 802.11 radio access technologies so they can communicate with each other and access internet ubiquitously. Another heterogeneous architecture that integrates IEEE 802.16 radio access technology and ad hoc communication between vehicles was proposed in [53]. This study supports public safety applications and Internet access. 


\begin{tabular}{|c|c|c|c|c|c|c|}
\hline $\begin{array}{l}\text { Beaconing } \\
\text { Approaches }\end{array}$ & Classification & Objective & Summary & Simulator & Vehicular Scenario & Simulation Results \\
\hline Guan et al. [13] & $\begin{array}{l}\text { Transmission power } \\
\text { adaptation }\end{array}$ & $\begin{array}{l}\text { Fulfilling reliability } \\
\text { and delay sensitive } \\
\text { active safety } \\
\text { applications }\end{array}$ & $\begin{array}{l}\text { Power control } \\
\text { information is } \\
\text { piggybacked in the } \\
\text { beacon header }\end{array}$ & $\begin{array}{l}\text { OPNET } \\
\text { simulator }\end{array}$ & $\begin{array}{l}\text { Highway vehicular } \\
\text { scenario }\end{array}$ & $\begin{array}{l}\text { Their algorithm performs better in terms of } \\
\text { message loss probability as compared to the } \\
\text { no-power control algorithm }\end{array}$ \\
\hline Artimy et al. [36] & $\begin{array}{l}\text { Transmission power } \\
\text { adaptation }\end{array}$ & $\begin{array}{l}\text { Maintaining } \\
\text { connectivity }\end{array}$ & $\begin{array}{l}\text { local vehicles traffic } \\
\text { density estimation }\end{array}$ & $\begin{array}{l}\text { Self build } \\
\text { simulator }\end{array}$ & $\begin{array}{l}\text { highway vehicular } \\
\text { scenario }\end{array}$ & $\begin{array}{l}\text { Simulation shows dynamic transmission } \\
\text { range achieves optimal connectivity }\end{array}$ \\
\hline $\begin{array}{l}\text { Torrent-Moreno et } \\
\text { al. [4] }\end{array}$ & $\begin{array}{l}\text { Transmission power } \\
\text { adaptation }\end{array}$ & $\begin{array}{l}\text { Reducing beacon load } \\
\text { and fast message } \\
\text { dissemination }\end{array}$ & $\begin{array}{l}\text { Prioritize emergency } \\
\text { messages and receiver } \\
\text { based relay self election }\end{array}$ & ns-2 simulator & $\begin{array}{l}\text { Realistic highway } \\
\text { scenario }\end{array}$ & $\begin{array}{l}\text { Their protocol performs better in terms of } \\
\text { channel access time and probability of } \\
\text { message reception as compared to the } \\
\text { no-power control algorithm }\end{array}$ \\
\hline Samara et al. [37] & $\begin{array}{l}\text { Transmission power } \\
\text { adaptation }\end{array}$ & $\begin{array}{l}\text { High message } \\
\text { reliability and } \\
\text { reducing channel } \\
\text { congestion }\end{array}$ & $\begin{array}{l}\text { A vehicle counts the } \\
\text { number of beacons lost } \\
\text { or successfully received } \\
\text { in } 1 \text { second }\end{array}$ & $\begin{array}{l}\text { Numerical } \\
\text { analysis }\end{array}$ & $\begin{array}{l}\text { Highway mobility } \\
\text { scenario }\end{array}$ & $\begin{array}{l}\text { Their algorithm adjusts transmission power } \\
\text { with respect to the channel congestion status }\end{array}$ \\
\hline Rawat et al. [15] & $\begin{array}{l}\text { Hybrid transmission } \\
\text { power and contention } \\
\text { window adaptation }\end{array}$ & $\begin{array}{l}\text { High reliability and } \\
\text { low dissemination } \\
\text { delay }\end{array}$ & $\begin{array}{l}\text { local vehicles density } \\
\text { estimation and } \\
\text { adjustment of } \\
\text { contention window }\end{array}$ & ns-2 simulator & $\begin{array}{l}\text { Highway mobility } \\
\text { scenario }\end{array}$ & $\begin{array}{l}\text { Proposed algorithm performs better in terms } \\
\text { of throughput and average delay as } \\
\text { compared to the default EDCA algorithm }\end{array}$ \\
\hline Kayhan et al. [9] & $\begin{array}{l}\text { Beacon rate } \\
\text { adaptation }\end{array}$ & $\begin{array}{l}\text { Reducing congestion } \\
\text { and increasing } \\
\text { message reception }\end{array}$ & $\begin{array}{l}\text { Adaptation based on } \\
\text { emergency status and } \\
\text { percentage of } \\
\text { directional vehicles }\end{array}$ & $\begin{array}{l}\text { JiST/SWANs } \\
\text { simulator }\end{array}$ & $\begin{array}{l}\text { realistic urban } \\
\text { environment }\end{array}$ & $\begin{array}{l}\text { Proposed algorithm performs better in terms } \\
\text { of probability of awareness, beaconing load } \\
\text { and average delay as compared to the fixed } \\
\text { beaconing algorithm }\end{array}$ \\
\hline Schmidt et al. [8] & $\begin{array}{l}\text { Beacon rate } \\
\text { adaptation }\end{array}$ & $\begin{array}{l}\text { Compromise between } \\
\text { information accuracy } \\
\text { and bandwidth } \\
\text { consumption }\end{array}$ & $\begin{array}{l}\text { Vehicular traffic } \\
\text { behaviour }\end{array}$ & None & None & $\begin{array}{l}\text { Their analysis shows that vehicle's position } \\
\text { error and beaconing load depends on beacon } \\
\text { rate and velocity/density }\end{array}$ \\
\hline Sommer et al. [12] & $\begin{array}{l}\text { Beacon rate } \\
\text { adaptation }\end{array}$ & $\begin{array}{l}\text { achieving congestion } \\
\text { aware and low latency } \\
\text { wireless channel }\end{array}$ & $\begin{array}{l}\text { channel quality and } \\
\text { importance of the } \\
\text { message }\end{array}$ & $\begin{array}{l}\text { OMNeT++ } \\
\text { simulator }\end{array}$ & $\begin{array}{l}\text { realistic urban } \\
\text { environment }\end{array}$ & $\begin{array}{l}\text { The proposed algorithm achieves larger } \\
\text { distance message dissemination as } \\
\text { compared to the flooding scheme }\end{array}$ \\
\hline Djahel et al. [45] & $\begin{array}{l}\text { Hybrid beacon and } \\
\text { transmission power } \\
\text { adaptation }\end{array}$ & $\begin{array}{l}\text { Achieving congestion } \\
\text { control and message } \\
\text { reception }\end{array}$ & $\begin{array}{l}\text { Beacon Reception rate, } \\
\text { packet collision rate } \\
\text { and medium busy time }\end{array}$ & OPNET & $\begin{array}{l}\text { Highway vehicular } \\
\text { environment }\end{array}$ & $\begin{array}{l}\text { Their algorithm performs better in terms of } \\
\text { total delay, beacons delivery ratio and } \\
\text { emergency message reception ratio as } \\
\text { compared to the off-congestion control } \\
\text { algorithm }\end{array}$ \\
\hline Huang et al. [47] & $\begin{array}{l}\text { Hybrid beacon and } \\
\text { transmission power } \\
\text { adaptation }\end{array}$ & $\begin{array}{l}\text { Maintaining accurate } \\
\text { positional information } \\
\text { and avoiding control } \\
\text { channel congestion }\end{array}$ & $\begin{array}{l}\text { Tracking positional } \\
\text { error difference }\end{array}$ & $\begin{array}{l}\text { OPNET } \\
\text { simulator }\end{array}$ & $\begin{array}{l}\text { Realistic } \\
\text { microscopic traffic } \\
\text { simulator }\end{array}$ & $\begin{array}{l}\text { The results shows that the proposed design } \\
\text { reduces tracking error as compared to the } \\
\text { de-facto fixed algorithm }\end{array}$ \\
\hline
\end{tabular}




\section{Research Directions and Open Issues}

The state of the arts beaconing solutions is reviewed, compared and criticized. These solutions can be counted as a basement of the beaconing in vehicular environments. However, yet they do not address many open issues. The following are some of the research directions.

Multi-hop beaconing in urban environments: In urban areas, there are different types of radio obstacles such as trucks (moving obstacles) and buildings (static obstacles) where a single hop beaconing can not penetrate them. Thus, multi-hop beaconing might be necessary in non-line of sight areas.

Traffic-aware and network-aware beaconing approaches: Beaconing frequency adaptation is a challenging future research work. The vehicular traffic situations and network load might be considered to tune the duty cycle of beaconing generation. In this case, the compromise of beaconing load and accuracy of positioning requirements should be taken into consideration in response with heterogeneity of vehicular environments.

Realistic Vehicular Network Scenarios: As witnessed from the state of the art described in earlier sections, most of the novel beaconing solutions were simulated in unrealistic vehicular scenarios, i.e., a vehicular scenario without considering short-term and long-term fading. The difference between simulation experiments using realistic and unrealistic vehicular topologies may result in the expense of human lives which is not affordable. Therefore, the existing research works need to be validated using realistic vehicular scenarios.

Forwarding optimizations: Most of the reviewed routing solutions considered unit disc transmission range, which does not hold true in the realistic vehicular environment as shadowing and different types of fading affects the radio propagation. Thus, it is necessary to utilize packet forwarding optimization by considering Quality of Service (QoS) during packet routing.

Multi-hop Beaconing in multi-radio enabled vehicular networks: Next Generation Network (NGN) aims to integrate different radio access technologies in order to provide seamless mobility and QoS at anywhere and anytime. Thus, it is crucial to design efficient adaptive beaconing algorithms over different wireless access technologies (WiFi, WiMAX, LTE) and decision for optimal selection between them in heterogeneous vehicular networks.

Beaconing in infrastructure-based vehicular networks: The implementation cost of cellular communication systems is high compared with infrastructure-based vehicular networks. In vehicular networks, the access points can be used as an intermediate node to relay data packets to other vehicles in multi-hop fashion. Thus, designing efficient routing in this environment can be used in various applications such as e-commerce, Electronic Toll Collection (ETC) and road-side advertisement services.

\section{Conclusions}

In this article, we summarized several types of adaptive beaconing approaches that either specifically proposed or adapted for the vehicular environments. From this survey perspective, it is observed that much endeavours have been made to develop efficient adaptive beaconing approaches. However, as can be seen in Table 1, there are pitfalls in the comparative study for most beaconing approaches since they only consider either mathematical analysis or simulation study. Also, so far none of reviewed beaconing solutions attempt real test-bed implementation. It is worth mentioning that simulation, practical implementation and mathematical modeling are three complementary pillars to give creditability of designed beaconing approaches since real world implementation verifies the assumptions made in the simulation and mathematical analysis phase. Moreover, there is no a unified solution or a standard benchmark for performance evaluation of beaconing approaches. Thus, solving the aforementioned issues and developing a benchmark beaconing solution for real world evaluation purpose are worth the effort in the future research opportunities. 


\section{References}

1. ITS-Standards, Intelligent transportation systems, U.S. Department of Transportation, http://www.standards.its.dot.gov/about.asp (1996)

2. L. Cheng, B. Henty, D. Stancil, F. Bai, P. Mudalige, Mobile vehicle-to-vehicle narrow-band channel measurement and characterization of the $5.9 \mathrm{ghz}$ dedicated short range communication (dsrc) frequency band, IEEE Transactions on Selected Areas in Communications 25 (8) (2007) 1501-1516. IEEE.

3. E. van Eenennaam, K. Wolterink, G. Karagiannis, G. Heijenk, Exploring the solution space of beaconing in vanets, in: Proceeding of the 2009 IEEE International Vehicular Networking Conference, Tokyo: IEEE, 2009, pp. 1-8.

4. M. Torrent-Moreno, Inter-vehicle communications: assessing information dissemination under safety constraints, in: Proceedings of the 2007 IEEE Conference Wireless on Demand Network Systems and Services, Austria: IEEE, 2007, pp. 59-64.

5. J. Lloret, A. Canovas, A. Catalá, M. Garcia, Group-based protocol and mobility model for vanets to offer internet access, Journal of Network and Computer Applications DOI: 10.1016 j.jnca.2012.02.009 (2012) 2224 - 2245. Elsevier.

6. J. Nzouonta, N. Rajgure, G. Wang, C. Borcea, Vanet routing on city roads using real-time vehicular traffic information, IEEE Transactions on Vehicular Technology 58 (7) (2009) 3609 - 3626. IEEE.

7. R. Fukui, H. Koike, H. Okada, Dynamic integrated transmission control(ditrac) over inter-vehicle communications in its, in: Proceedings of the 2002 IEEE Vehicular Technology Conference, Birmingham: IEEE, 2002, pp. $483-487$.

8. R. Schmidt, T. Leinmuller, E. Schoch, F. Kargl, G. Schafer, Exploration of adaptive beaconing for efficient intervehicle safety communication, IEEE Network 24 (1) (2010) 14 - 19. IEEE.

9. K. Ghafoor, K. Bakar, E. van Eenennaam, R. Khokhar, A. Gonzalez, A fuzzy logic approach to beaconing for vehicular ad hoc networks, Accepted for publication in Telecommunication Systems Journal.

10. K. Ghafoor, K. Bakar, H. AL Hashimi, K. Lee, A novel delay and reliability aware inter vehicle routing protocol, Network Protocols and Algorithms 2 (2) (2010) 66 - 88. Macrothink Institute.

11. J. Mittag, F. Thomas, J. Härri, H. Hartenstein, A comparison of single-and multi-hop beaconing in vanets, in: Proceedings of the 2009 ACM International Workshop on VehiculAr InterNETworking, Beijing: ACM, 2009, pp. 69-78.

12. C. Sommer, O. Tonguz, F. Dressler, Adaptive beaconing for delay-sensitive and congestion-aware traffic information systems, in: Proceedings of the 2010 IEEE International Vehicular Networking Conference (VNC), Jersey: IEEE, 2010 , pp. 1 -8 .

13. X. Guan, R. Sengupta, H. Krishnan, F. Bai, A feedback-based power control algorithm design for vanet, in: Proceedings of the 2007 IEEE International Conference on Mobile Networking for Vehicular Environments, USA: IEEE, 2007, pp. $67-72$.

14. H. AL-Hashimi, K. Bakar, K. Ghafoor, Inter-domain proxy mobile ipv6 based vehicular network, Network Protocols and Algorithms 2 (4) (2011) 1-15. MacroThink.

15. D. Rawat, D. Popescu, G. Yan, S. Olariu, Enhancing vanet performance by joint adaptation of transmission power and contention window size, Transactions on Parallel and Distributed Systems 22 (9) (2011) 1528 - 1535. IEEE.

16. European-ITS, Eits-technical report 102638 v1.1.1, European Telecommunications Standards Institute (ETSI), http://www.etsi.org/WebSite/homepage.aspx (2009).

17. I. NHTSA, Joint program office," report to congress on the national highway traffic safety administration its program, program progress during 1992-1996 and strategic plan for 1997-2002, US Department of Transportation, Washington, DC.

18. D. Godbole, R. Sengupta, J. Misener, N. Kourjanskaia, J. Michael, Benefit evaluation of crash avoidance systems, Transportation Research 1621 (1) (1998) 1 - 9. Trans. Res. Board.

19. R. Reinders, M. van Eenennaam, G. Karagiannis, G. Heijenk, Contention window analysis for beaconing in vanets, in: Proceedings of the 2011 IEEE International Conference on Wireless Communications and Mobile Computing (IWCMC), Istanbul: IEEE, 2004, pp. $1481-1487$.

20. L. Yang, J. Guo, Y. Wu, Channel adaptive one hop broadcasting for vanets, in: Proceedings of the 2008 IEEE International Conference on Intelligent Transportation Systems, Beijing : IEEE, 2008, pp. 369-374.

21. Y. Tseng, S. Ni, Y. Chen, J. Sheu, The broadcast storm problem in a mobile ad hoc network, Wireless Networks 8 (2) (2002) 153 - 167. IEEE.

22. E. M. van Eenennaam, G. Karagiannis, G. Heijenk, Towards scalable beaconing in vanets, in: Proceedings of the 2010 ERCIM Workshop on eMobility, Lulea: Lulea University of Technology, 2010, pp. 103-108.

23. F. Ros, P. Ruiz, I. Stojmenovic, Acknowledgment-based broadcast protocol for reliable and efficient data dissemination in vehicular ad-hoc networks, IEEE Transactions on Mobile Computing 11 (1) (2012) 33 - 46. IEEE.

24. M. Torrent-Moreno, P. Santi, H. Hartenstein, Distributed fair transmit power adjustment for vehicular ad hoc networks, in: Proceedings of the 2007 IEEE International Confgerence on Sensor and Ad Hoc Communications and Networks, Reston, VA: IEEE, 2006, pp. 479-488.

25. M. Artimy, Local density estimation and dynamic transmission-range assignment in vehicular ad hoc networks, IEEE Transactions on Intelligent Transportation Systems 8 (3) (2007) 400 - 412. IEEE.

26. G. Samara, S. Ramadas, W. Al-Salihy, Safety Message Power Transmission Control for Vehicular Ad hoc Networks, Computer Science 6 (10) (2010) 1027 - 1032. Science Publications.

27. G. Caizzone, P. Giacomazzi, L. Musumeci, G. Verticale, A power control algorithm with high channel availability for vehicular ad hoc networks, in: Proceedings of the 2005 IEEE International Conference on Communications, Seoul: IEEE, 2005 , pp. $3171-3176$.

28. M. Torrent-Moreno, P. Santi, H. Hartenstein, Vehicle-to-vehicle communication: Fair transmit power control for safety critical information, IEEE Transaction for Vehicular Technology 58 (7) (2009) 3684 - 3703 . IEEE.

29. M. Torrent-Moreno, F. Schmidt-Eisenlohr, H. Fubler, H. Hartenstein, Effects of a realistic channel model on packet forwarding in vehicular ad hoc networks, in: Proceedings of the 2007 IEEE Conference on Wireless Communications and Networking, USA: IEEE, 2006, pp. 385-391. 
30. NS, Network simulator, http://nsnam.isi.edu/nsnam/index.php/Main Page (June 2011).

31. M. Nakagami, The m-distribution-A general formula of intensity distribution of rapid fadinge. W. C. Hoffman. ed., Statistical Method of Radio Propagation, Pergamon Press, 1960.

32. S. Narayanaswamy, V. Kawadia, R. Sreenivas, P. Kumar, Power control in ad-hoc networks: Theory, architecture, algorithm and implementation of the compow protocol, in: Proceedings of the 2002 European Wireless Conference Next Generation Wireless Networks: Technologies, protocols, Services and Applications, Italy: European Wireless, 2002, pp. 1 - 6.

33. P. Cheng, K. Lee, M. Gerla, J. Harri, Geodtn+ nav: Geographic dtn routing with navigator prediction for urban vehicular environments, Mobile Networks and Applications 15 (1) (2010) 61 - 82. Kluwer Academic Publishers.

34. J. Gomez, A. Campbell, A case for variable-range transmission power control in wireless multihop networks, in: Proceedings Twenty-third AnnualJoint Conference of the IEEE Computer and Communications Societies, Hong kong: IEEE, 2004, pp. 1425-1436.

35. R. Ramanathan, R. Rosales-Hain, Topology control of multihop wireless networks using transmit power adjustment, in: Proceedings Nineteenth Annual Joint Conference of the IEEE Computer and Communications Societies, Hong kong: IEEE, 2000, pp. 404-413.

36. M. Artimy, W. Robertson, W. Phillips, Assignment of dynamic transmission range based on estimation of vehicle density, in: Proceedings of the 2nd ACM international workshop on Vehicular ad hoc networks, Germany: ACM, 2005 , pp. 40-48.

37. G. Samara, S. Ramadas, W. Al-Salihy, Safety message power transmission control for vehicular ad hoc networks, Computer Science 6 (10) (2010) 1027 - 1032. Kluwer Academic Publishers.

38. S. Rezaei, R. Sengupta, H. Krishnan, X. Guan, P. Student, Adaptive communication scheme for cooperative active safety system (2008).

39. S. Rezaei, R. Sengupta, H. Krishnan, X. Guan, Reducing the communication required by dsrc-based vehicle safety systems, in: Proceedings of the 2007 IEEE International Conference on Intelligent Transportation Systems, Bellevue, WA: IEEE, 2007, pp. 361-366.

40. C. Sommer, O. Tonguz, F. Dressler, Traffic information systems: efficient message dissemination via adaptive beaconing, IEEE Communications Magazine 49 (5) (2011) 173 - 179. IEEE.

41. C. Thaina, K. Nakorn, K. Rojviboonchai, A study of adaptive beacon transmission on vehicular ad-hoc networks, in: Proceeding of the 2011 IEEE 13th International Conference on Communication Technology (ICCT), Vancouver: ACM, 2011, pp. 597-602.

42. A. Boukerche, C. Rezende, R. Pazzi, Improving neighbor localization in vehicular ad hoc networks to avoid overhead from periodic messages, in: Proceeding of the 2009 IEEE Global Telecommunications Conference, GLOBECOM, USA: IEEE, 2009, pp. 1-6.

43. F. Bai, N. Sadagopan, A. Helmy, Important: A framework to systematically analyze the impact of mobility on performance of routing protocols for adhoc networks, in: Proceedings of the 2003 22th Annual Joint Conference of the IEEE Computer and Communications., USA: IEEE, 2008, pp. 825 - 835.

44. H. Nguyen, A. Bhawiyuga, H. Jeong, A comprehensive analysis of beacon dissemination in vehicular networks, in: Proceedings of the 75th IEEE Vehicular Technology Conference, Korea: IEEE, 2012, pp. 1 - 5.

45. S. Djahel, Y. Ghamri-Doudane, A robust congestion control scheme for fast and reliable dissemination of safety messages in vanets, in: Proceeding of the 2012 IEEE Conference Wireless Communications and Networking, Paris, France: IEEE, 2012, pp. 2264-2269.

46. O. Technologies, Opnet modeler, http://www.opnet.com/. (Augast 2012).

47. C. Huang, Y. Fallah, R. Sengupta, H. Krishnan, Adaptive intervehicle communication control for cooperative safety systems, IEEE Network 24 (1) (2010) 6 - 13. IEEE.

48. OPNET, Opnet modeler, http://www.opnet.com/ (June 2012).

49. B. Kerner, The physics of traffic: empirical freeway pattern features, engineering applications, and theory, Springer Verlag, 2004.

50. A. Vinel, V. Vishnevsky, Y. Koucheryavy, A simple analytical model for the periodic broadcasting in vehicular ad-hoc networks, in: Proceedings of the 2008 IEEE International GLOBECOM Workshops, Philadelphia, PA: IEEE, 2008, pp. 1 -5 .

51. N. Mariyasagayam, H. Menouar, M. Lenardi, An adaptive forwarding mechanism for data dissemination in vehicular networks, in: Proceedings of the 2009 IEEE Vehicular Networking Conference, Boston: IEEE, 2009, pp. $1-5$.

52. C. Hung, H. Chan, E. Wu, Mobility pattern aware routing for heterogeneous vehicular networks, in: Proceedings of the 2008 International Conference on Wireless Communications and Networking, Las Vegas: IEEE, 2008, pp. $2200-2205$.

53. K. Yang, S. Ou, H. Chen, J. He, A multihop peer-communication protocol with fairness guarantee for ieee 802.16-based vehicular networks, IEEE Transactions on Vehicular Technology 56 (6) (2007) 3358 - 3370 . IEEE.

54. I. Lequerica, P. Ruiz, V. Cabrera, Improvement of vehicular communications by using $3 \mathrm{~g}$ capabilities to disseminate control information, IEEE Network Magazine 24 (1) (2010) 32 - 38. IEEE.

55. D. Oh, P. Kim, J. Song, S. Jeon, H. Lee, Design considerations of satellite-based vehicular broadband networks, IEEE Wireless Communications Magazine 12 (5) (2005) 91 - 97. IEEE.

56. Y. Ko, M. Sim, M. Nekovee, Wi-fi based broadband wireless access for users on the road, BT Technology Journal 24 (2) (2006) 123 - 129. Springer. 